\title{
The association between lower Hounsfield units of the upper instrumented vertebra and proximal junctional kyphosis in adult spinal deformity surgery with a minimum 2-year follow-up
}

\author{
Ping-Guo Duan, MD, ${ }^{1,3}$ Praveen V. Mummaneni, MD, ${ }^{1}$ Joshua Rivera, BA, ${ }^{1}$ \\ Jeremy M. V. Guinn, BS, ${ }^{1}$ Minghao Wang, MD, ${ }^{1}$ Zhuo Xi, MD, ${ }^{1}$ Bo Li, MD, ${ }^{1}$ Hao-Hua Wu, MD, ${ }^{2}$ \\ Christopher P. Ames, MD, ${ }^{1}$ Shane Burch, MD, ${ }^{2}$ Sigurd H. Berven, MD, ${ }^{2}$ and Dean Chou, MD ${ }^{1}$ \\ Departments of ${ }^{1}$ Neurological Surgery and ${ }^{2}$ Orthopaedic Surgery, University of California, San Francisco, California; and \\ ${ }^{3}$ Department of Orthopaedic Surgery, The First Affiliated Hospital of Nanchang University, Nanchang, People's Republic of China
}

\begin{abstract}
OBJECTIVE Patients undergoing long-segment fusions from the lower thoracic (LT) spine to the sacrum for adult spinal deformity (ASD) correction are at risk for proximal junctional kyphosis (PJK). One mechanism of PJK is fracture of the upper instrumented vertebra (UIV) or higher (UIV+1), which may be related to bone mineral density (BMD). Because Hounsfield units (HUs) on CT correlate with BMD, the authors evaluated whether HU values were correlated with PJK after long fusions for ASD.
\end{abstract}

METHODS The authors performed a retrospective study of patients older than 50 years who had undergone ASD correction from the LT spine to the sacrum in the period from October 2007 to January 2018 and had a minimum 2-year follow-up. Demographic and spinopelvic parameters were measured. HU values were measured on preoperative CT at the UIV, UIV+1, and UIV+2 (2 levels above the UIV) levels and were assessed for correlations with PJK.

RESULTS The records of 127 patients were reviewed. Fifty-four patients (19 males and 35 females) with a mean age of 64.91 years and mean follow-up of 3.19 years met the study inclusion criteria; there were 29 patients with PJK and 25 patients without. There was no statistically significant difference in demographics or follow-up between these two groups. Neither was there a difference between the groups with regard to postoperative pelvic incidence (PI), sacral slope (SS), lumbar lordosis (LL), PI minus LL (PI-LL), thoracic kyphosis (TK), or sagittal vertical axis (SVA; all $p>0.05)$. Postoperative pelvic tilt $(p=0.003)$ and T1 pelvic angle $(p=0.014)$ were significantly higher in patients with PJK than in those without. Preoperative HUs at UIV, UIV+1, and UIV+2 were 120.41, 124.52, and 129.28 in the patients with PJK, respectively, and $152.80,155.96$, and 160.00 in the patients without PJK, respectively ( $p=0.011,0.02$, and 0.018$)$. Three receiver operating characteristic $(\mathrm{ROC})$ curves for preoperative $\mathrm{HU}$ values at the UIV, UIV+1, and UIV+2 as a predictor for PJK were established, with areas under the ROC curve of 0.710 (95\% Cl 0.574-0.847), $0.679(95 \% \mathrm{Cl} 0.536-0.821)$, and 0.681 (95\% Cl 0.539-0.824), respectively. The optimal HU value by Youden index was $104 \mathrm{HU}$ at the UIV (sensitivity 0.840 , specificity 0.517 ), $113 \mathrm{HU}$ at the UIV+1 (sensitivity 0.720 , specificity 0.517 ), and $110 \mathrm{HU}$ at the UIV+2 (sensitivity 0.880 , specificity 0.448 ).

CONCLUSIONS In patients undergoing long-segment fusions from the LT spine to the sacrum for ASD, PJK was associated with lower HU values on CT at the UIV, UIV+1, and UIV+2. The measurement of HU values on preoperative CTs may be a useful adjunct for ASD surgery planning.

https://thejns.org/doi/abs/10.3171/2020.5.FOCUS20192

KEYWORDS proximal junctional kyphosis; PJK; upper instrumented vertebra; UIV; bone mineral density; Hounsfield units

ABBREVIATIONS ASD = adult spinal deformity; $A U C=$ area under the ROC curve; $B M D=$ bone mineral density; $C S V L=$ central sacral vertical line; $D X A=$ dual-energy x-ray absorptiometry; $\mathrm{HU}=$ Hounsfield unit; $\mathrm{LL}=$ lumbar lordosis; $\mathrm{LT}=$ lower thoracic; $\mathrm{PI}=$ pelvic incidence; PI-LL = PI minus LL; $\mathrm{PJK}=$ proximal junctional kyphosis; $\mathrm{PT}=$ pelvic tilt; ROC = receiver operating characteristic; $\mathrm{ROI}=$ region of interest; $\mathrm{SS}=$ sacral slope; SVA = sagittal vertical axis; TK = thoracic kyphosis; TPA = T1 pelvic angle; UIV = upper instrumented vertebra; UIV+1 = 1 level above the UIV; UIV+2 = 2 levels above the UIV.

SUBMITTED March 4, 2020. ACCEPTED May 13, 2020.

INCLUDE WHEN CITING DOI: 10.3171/2020.5.FOCUS20192. 
$\mathrm{T}$ HE treatment of adult spinal deformity (ASD) has become more prevalent, with ASD affecting up to $60 \%$ of the elderly population..$^{1,2}$ Despite advances in the surgical treatment of ASD, proximal junctional kyphosis (PJK) is a well-recognized postoperative risk. ${ }^{3-7}$ The prevalence of PJK in ASD patients undergoing longsegment fusions has been reported to be between $5.8 \%$ and $62 \% .^{3,5,8-12}$ Multiple studies on PJK have been performed, with more and more evidence showing that PJK appears to be a multifactorial phenomenon..$^{3,6,10,11,13-17}$ One mechanism of PJK is fracture of the upper instrumented vertebra (UIV) or the level above (UIV+1). ${ }^{3,13,14,18}$ Maruo et al. reported that fracture at the UIV was the most common mechanism for PJK, especially in the lower thoracic (LT) spine. ${ }^{3}$ It has also been reported that low bone mineral density (BMD) is a potential risk factor for PJK. ${ }^{18-20}$ Furthermore, other studies have reported that even osteopenia may be a factor in vertebral body fracture, subluxation, and PJK. ${ }^{6,21}$

BMD is normally evaluated using dual-energy x-ray absorptiometry (DXA). ${ }^{18,22}$ However, DXA scans in the lumbar spine may not always be accurate given the amount of degeneration obscuring the true values, and spinal instrumentation can obfuscate interpretation. ${ }^{18,22}$ Moreover, not all patients obtain a preoperative DXA scan. Hounsfield units (HUs) on CT as a proxy for BMD have been shown to correlate with DXA values. ${ }^{18,22-24}$ Studies have demonstrated a correlation between HUs and DXA scores when comparing osteoporotic and non-osteoporotic patients, ${ }^{23,25}$ with a positive correlation between the T-score on DXA and the HU value on spinal CT. ${ }^{18,25}$ However, these studies have focused on degenerative conditions of the lumbar spine, and it is unclear if HUs reliably reflect the BMD of the LT vertebrae, the often-chosen UIV level in ASD surgery. We evaluated whether the HU values at the UIV, UIV+1, and UIV+2 (2 levels above the UIV) were associated with PJK in ASD patients over 50 years old.

\section{Methods \\ Patient Cohort}

We performed a retrospective study of patients who had undergone ASD correction from the LT spine (T9-T12) as their UIV to the sacrum in the period from October 2007 to January 2018 at the University of California, San Francisco; four spine surgeons had performed the procedures. Patients had to meet at least one of the following inclusion criteria: age $>50$ years; scoliotic Cobb angle $>20^{\circ}$; sagittal vertical axis (SVA) $>5 \mathrm{~cm}$; pelvic tilt $(\mathrm{PT})>25^{\circ}$; minimum 2-year follow-up; preoperative CT that included the UIV, UIV+1, and UIV+2; and ASD fusion at $\geq 6$ levels for sagittal plane realignment. The majority of patients had a diagnosis of adult idiopathic scoliosis, adult degenerative scoliosis, or flat back deformity. Patients with tumor, trauma, or infection and/or without either long-standing cassette films or a 2-year follow-up were excluded.

\section{Radiographic Measurements}

All patients had 36-inch standing anteroposterior and lateral spinal radiographs preoperatively, within 1 month postoperatively, and at the last follow-up. Radiographic

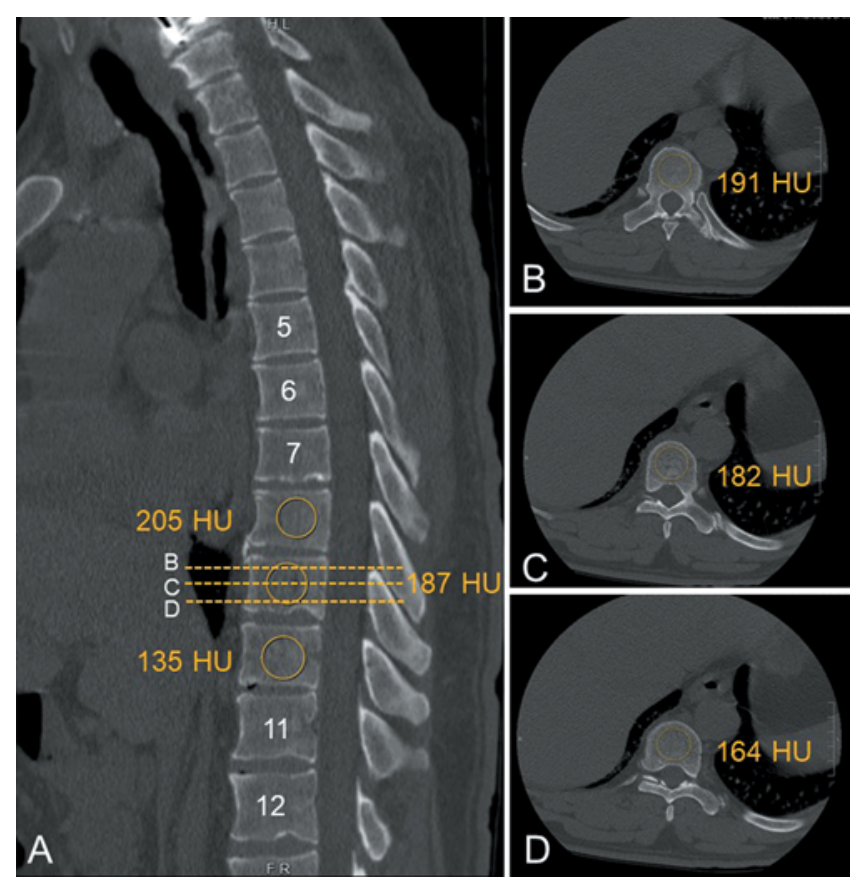

FIG. 1. Illustration of measuring $\mathrm{HU}$ values on preoperative $\mathrm{CT}$ at the UIV (T10 in this case), UIV+1 (T9), and UIV+2 (T8). The four separate measurements made are in the midsagittal plane (A), at the midbody axial plane $(\mathbf{C})$, in the axial plane just inferior to the superior endplate (B), and in the axial plane just superior to the inferior endplate (D).

spinopelvic parameters measured were as follows: pelvic incidence (PI), PT, lumbar lordosis (LL), PI minus LL (PI-LL), sacral slope (SS), T1 pelvic angle (TPA), T5-12 thoracic kyphosis (TK), SVA, central sacral vertical line (CSVL), and scoliotic Cobb angle.

\section{HU Measurements}

$\mathrm{HU}$ values were measured on preoperative CTs at the UIV, UIV+1, and UIV+2 according to the methodology described by Schreiber et al. ${ }^{25}$ According to this method, an ellipsoid region of interest (ROI) is used on the CT, ideally including the largest possible cancellous bone region while excluding cortical margins, osteophytes, and Schmorl nodes. The ROIs are measured in four separate sections of each vertebra: the midsagittal plane, immediately inferior to the superior endplate, in the middle of the vertebral body, and immediately superior to the inferior endplate. The HU value of each ROI is calculated using the PACS. The mean HU value of the four ROIs is defined as the vertebral body mean HU (Fig. 1).

\section{Proximal Junctional Kyphosis}

The proximal junctional angle (PJA) was defined as the sagittal Cobb angle between the inferior endplate of the UIV and the superior endplate of the UIV+2. Radiographic PJK was defined as PJA $\geq 10^{\circ}$ and at least $10^{\circ}$ greater than the corresponding preoperative measurement. ${ }^{8,26}$

\section{Statistical Analysis}

Continuous variables were presented as means with 
TABLE 1. Classification of PJK

\begin{tabular}{cc}
\hline Type of PJK & No. \\
\hline Type 1 & 11 \\
\hline Type 2 & 4 \\
\hline Type 3 & 14 \\
\hline
\end{tabular}

Type 1 = disc and ligament failure; type 2 = bone fracture; type 3 = implantbone interface failure.

standard deviations, and categorical variables were presented as frequencies and percentages. Pre- and postoperative continuous variables were analyzed with independent-samples t-tests. A $p$ value $<0.05$ was considered statistically significant. A receiver operating characteristic (ROC) curve was analyzed to establish separation criteria between patients with PJK and without PJK. The areas under the ROC curve (AUCs) were calculated for preoperative HU assessment at the UIV. The most appropriate threshold (cutoff value) of $\mathrm{HU}$ at the UIV with a higher sensitivity and specificity was also established using the ROC curve. Statistical analysis was performed using SPSS Statistics version 24.0 (IBM Corp.).

\section{Results}

After evaluating 127 patients who had LT to sacral fusions in the study period, we found a total of 54 patients (19 males and 35 females) who met the inclusion criteria (mean age $64.91 \pm 7.59$ years) and had a mean follow-up of $3.19 \pm 1.14$ years. There were 29 patients with PJK and 25 patients without; the overall rate of PJK was 53.7\% (29 of 54). Classification of PJK according to Yagi et al. revealed 11 type 1,4 type 2 , and 14 type 3 cases (Table 1$).{ }^{15} \mathrm{We}$ also categorized the patients using an HU threshold of 110 since that value is the threshold for osteoporotic spines. With this threshold, $73.9 \%$ (17 of 23) of patients with HUs lower than 110 had PJK, and 38.7\% (12 of 31) of patients with HUs higher than 110 had PJK $(p=0.014)$. Prophylactic methylmethacrylate augmentation was performed at the UIV or UIV+1 in 19 patients. Of these, 10 had PJK, and 9 did not $(\mathrm{p}=0.781)$. Only 2 patients had interspinous tethers, and no patients had transitional rods to the UIV. There was no difference in age, BMI, sex, and follow-up between the two groups.

There was no statistical difference between the groups with regard to preoperative PI, SS, PT, LL, PI-LL, TK, TPA, SVA, CSVL, or Cobb angle (all p > 0.05; Table 2). Preoperative HUs at UIV, UIV+1, and UIV+2 were 120.41 $\pm 45.67,124.52 \pm 45.17$, and $129.28 \pm 45.99$ in patients with PJK, respectively, and $152.80 \pm 43.99,155.96 \pm 50.87$, and $160.00 \pm 46.31$ in the patients without PJK $(\mathrm{p}=0.011,0.02$, and 0.018 , respectively). Postoperative PT and TPA were significantly higher in patients with PJK than in those without ( $p=0.003$ and 0.014 , respectively). However, there was no statistical difference between the groups with regard to postoperative SS, LL, PI-LL, TK, SVA, and CSVL (all p > 0.05; Table 3). Multivariate regression analysis was performed, and there was no statistical correlation with PJK and other common causes of PJK (Table 4).
TABLE 2. Comparison of preoperative parameters in patients with and without PJK

\begin{tabular}{|c|c|c|c|}
\hline Parameter & PJK $(n=29)$ & No PJK $(n=25)$ & $p$ Value \\
\hline Age in yrs & $65.18 \pm 8.19$ & $64.64 \pm 7.20$ & 0.801 \\
\hline $\mathrm{BMI}$ in $\mathrm{kg} / \mathrm{m}^{2}$ & $26.99 \pm 5.34$ & $29.49 \pm 5.01$ & 0.105 \\
\hline $\mathrm{SS}^{\circ} \mathrm{in}^{\circ}$ & $32.96 \pm 11.32$ & $33.44 \pm 14.55$ & 0.895 \\
\hline PT in ${ }^{\circ}$ & $29.00 \pm 7.30$ & $26.76 \pm 14.32$ & 0.476 \\
\hline $\mathrm{Pl}$ in $^{\circ}$ & $61.56 \pm 11.74$ & $56.72 \pm 13.91$ & 0.180 \\
\hline LL in ${ }^{\circ}$ & $36.48 \pm 15.99$ & $31.36 \pm 17.34$ & 0.273 \\
\hline $\mathrm{Pl}-\mathrm{LL}$ in ${ }^{\circ}$ & $25.59 \pm 13.57$ & $25.36 \pm 14.47$ & 0.953 \\
\hline TPA in $^{\circ}$ & $29.85 \pm 8.23$ & $25.88 \pm 9.99$ & 0.123 \\
\hline TK in $^{\circ}$ & $30.07 \pm 13.74$ & $23.80 \pm 13.95$ & 0.109 \\
\hline SVA in $\mathrm{cm}$ & $8.71 \pm 5.45$ & $9.24 \pm 5.10$ & 0.726 \\
\hline CSVL in mm & $2.78 \pm 2.51$ & $2.33 \pm 1.40$ & 0.442 \\
\hline Cobb angle in ${ }^{\circ}$ & $28.07 \pm 18.04$ & $20.75 \pm 18.76$ & 0.162 \\
\hline HU at UIV & $120.41 \pm 45.67$ & $152.80 \pm 43.99$ & $0.011^{*}$ \\
\hline $\mathrm{HU}$ at $\mathrm{UIV}+1$ & $124.52 \pm 5.17$ & $155.96 \pm 50.87$ & $0.02^{*}$ \\
\hline $\mathrm{HU}$ at $\mathrm{UIV+2}$ & $129.28 \pm 45.99$ & $160.00 \pm 46.31$ & $0.018^{*}$ \\
\hline
\end{tabular}

$\mathrm{n}=$ number of patients.

${ }^{*} p<0.05$.

Three ROC curves for preoperative $\mathrm{HU}$ values at the $\mathrm{UIV}, \mathrm{UIV}+1$, and UIV+2 as a predictor for PJK were established, with an AUC of 0.710 (95\% CI 0.574-0.847) for the UIV, 0.679 (95\% CI 0.536-0.821) for UIV+1, and 0.681 (95\% CI 0.539-0.824) for UIV+2. The optimal HU cutoff value determined by the Youden index was $104 \mathrm{HU}$ at the UIV (sensitivity 0.840 , specificity 0.517 ), $113 \mathrm{HU}$ at the $\mathrm{UIV+1}$ (sensitivity 0.720 , specificity 0.517 ), and $110 \mathrm{HU}$ at the UIV+2 (sensitivity 0.880 , specificity 0.448 ; Fig. 2).

\section{Discussion}

Given the various rates, mechanisms, risk factors, and treatment strategies for PJK, the complication probably has a multifactorial etiology. ${ }^{3,6,10-17}$ Fracture of the UIV or the UIV+1 has been shown to be one of the mechanisms of PJK. ${ }^{3,13,14,18}$ Indeed, Maruo et al. have reported that fracture at the UIV is the most common mechanism. ${ }^{3}$ Burch et

TABLE 3. Comparison of postoperative parameters in patients with and without PJK

\begin{tabular}{|c|c|c|c|}
\hline Parameter & PJK $(n=29)$ & No PJK $(n=25)$ & $p$ Value \\
\hline$S S$ in $^{\circ}$ & $34.81 \pm 9.47$ & $39.92 \pm 10.96$ & 0.084 \\
\hline $\mathrm{PT}$ in ${ }^{\circ}$ & $26.65 \pm 8.11$ & $19.38 \pm 8.31$ & $0.003^{*}$ \\
\hline $\mathrm{LL}^{\circ}{ }^{\circ}$ & $53.61 \pm 10.99$ & $53.45 \pm 15.77$ & 0.967 \\
\hline $\mathrm{PI}-\mathrm{LL}$ in $^{\circ}$ & $10.92 \pm 7.72$ & $8.72 \pm 8.73$ & 0.344 \\
\hline $\mathrm{TPA}^{\circ}{ }^{\circ}$ & $24.62 \pm 9.25$ & $17.79 \pm 9.74$ & $0.014^{*}$ \\
\hline $\mathrm{TK}^{\circ}$ in $^{\circ}$ & $36.97 \pm 10.99$ & $33.67 \pm 14.66$ & 0.354 \\
\hline SVA in $\mathrm{cm}$ & $5.66 \pm 4.48$ & $4.52 \pm 3.40$ & 0.313 \\
\hline CSVL in $\mathrm{mm}$ & $2.27 \pm 1.63$ & $2.05 \pm 1.68$ & 0.635 \\
\hline
\end{tabular}

${ }^{*} p<0.05$. 
TABLE 4. Multivariate regression analysis of the statistical contribution of other risk factors for PJK

\begin{tabular}{lcccc}
\hline & & \multicolumn{2}{c}{$95 \% \mathrm{Cl}$} & \\
\cline { 3 - 4 } Parameter & Exp(B) & Lower & Upper & p Value \\
\hline Age & 1.006 & 0.900 & 1.125 & 0.910 \\
\hline Sex & 0.483 & 0.051 & 4.566 & 0.526 \\
\hline BMI & 1.095 & 0.934 & 1.285 & 0.263 \\
\hline Postop SS & 0.941 & 0.183 & 4.835 & 0.942 \\
\hline Postop PT & 0.745 & 0.140 & 3.963 & 0.730 \\
\hline Postop PI & 1.185 & 0.240 & 5.843 & 0.835 \\
\hline Postop LL & 0.944 & 0.803 & 1.109 & 0.481 \\
\hline Postop PI-LL & 1.026 & 0.875 & 1.204 & 0.750 \\
\hline Postop SVA & 0.894 & 0.616 & 1.297 & 0.554 \\
\hline Postop CSVL & 1.047 & 0.561 & 1.956 & 0.885 \\
\hline Postop TPA & 1.005 & 0.743 & 1.358 & 0.976 \\
\hline Postop TK & 0.994 & 0.906 & 1.091 & 0.900 \\
\hline
\end{tabular}

al. have reported that the presence of osteoporosis, which reflects a deterioration of bone strength, not just density, is a key etiological factor for insufficiency fractures, PJK, pseudarthrosis, and screw pullout. ${ }^{27}$ Other studies have documented similar findings, concluding that age and low BMD are potential risk factors for postoperative PJK. ${ }^{18-20}$ In addition to its PJK risk, Yagi et al. reported that low BMD is also a significant risk factor for proximal junctional failure..$^{20}$ One reason for these failures may be that poor bone quality either compromises the interface between bone and instrumentation or is insufficiently strong to withstand the new forces of the spine against a long lever arm from a multilevel fusion. , $^{6,17,21}$

Because the high incidence of PJK has become a vexing problem for both the patient and the treatment team, surgeons are becoming more aware of the importance of assessing BMD prior to surgical correction of ASD. Although DXA is currently considered to be the gold standard to assess BMD and its values have been shown to correlate with fracture risk, ${ }^{22,25}$ DXA is not always part of the routine preoperative workup. ${ }^{22}$ Furthermore, DXA has been shown to underestimate the magnitude of bone loss in spinal vertebrae ${ }^{27}$ leading to false-negative rates of fracture risk. ${ }^{28}$ Burch et al. have shown that vertebral fractures are highly associated with CT-measured BMD and finite element-estimated vertebral bone strength more so than DXA-measured BMD. ${ }^{27}$ Hendrickson et al. have shown that CT provides better accuracy and excellent reproducibility for measuring BMD by using HU values. ${ }^{28}$ However, for ASD surgeries, many surgeons will obtain a preoperative $\mathrm{CT}$, not a DXA scan, and the HU values on the CT may be a proxy for BMD, saving both cost and time. ${ }^{22,25}$ Lewiecki et al. found a linear correlation between $\mathrm{HU}$ values and compressive strength of bone. ${ }^{29} \mathrm{HU}$ values can also be used to estimate regional bone strength, and measuring the HU value in the planned screw trajectory may predict the strength of the bone-screw interface prior to screw insertion. ${ }^{25}$ Furthermore, several studies have reported reasonably good correlation between HUs and DXA value ${ }^{18,25,30,31}$ and between HUs and osteoporosis. ${ }^{23,25,32}$ Thus, the use of HUs on CT as a proxy for a DXA scan may be a reasonable option in the preoperative workup, and formal DXA can be performed if the CT indicates probable poor bone quality. ${ }^{18,25,28}$ Although the spine is the ROI in terms of surgical planning, the hip also needs to be evaluated when obtaining a DXA scan because the spine DXA may not be accurate with degenerative change or deformity, common findings in older patients requiring spine surgery. Moreover, if the lumbar spine is evaluated with DXA, an accurate assessment should be performed from L1 to L4, and the thoracic spine is currently not a standard area to perform DXA. ${ }^{33}$
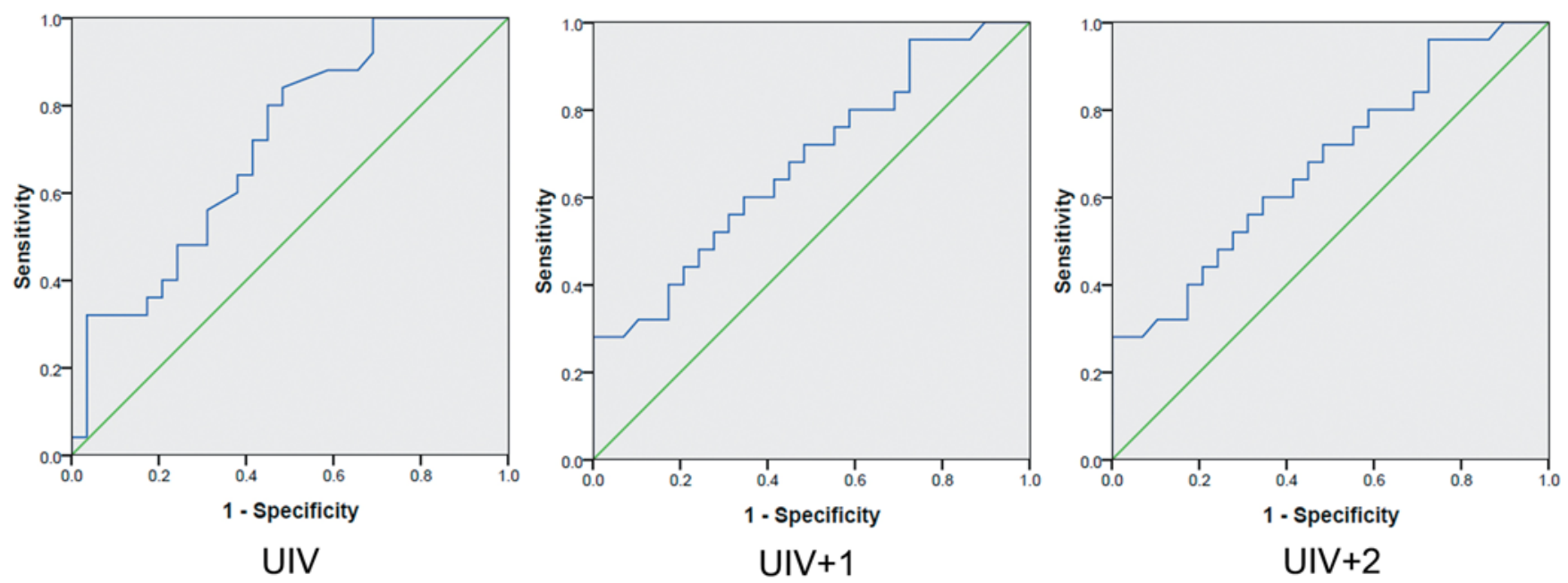

FIG. 2. Three ROC curves for preoperative HU values at the UIV, UIV+1, and UIV+2 as a predictor for PJK were established, with an AUC of $0.710(95 \% \mathrm{Cl} 0.574-0.847)$ for the UIV, $0.679(95 \% \mathrm{Cl} 0.536-0.821)$ for the UIV+1, and $0.681(95 \% \mathrm{Cl} 0.539-0.824)$ for the UIV+2. The optimal HU cutoff value determined by the Youden index was $104 \mathrm{HU}$ at the UIV (sensitivity 0.840 , specificity 0.517), $113 \mathrm{HU}$ at the UIV+1 (sensitivity 0.720 , specificity 0.517 ), and $110 \mathrm{HU}$ at the UIV+2 (sensitivity 0.880 , specificity 0.448 ). 
PJK has been defined as a sagittal Cobb angle of more than $10^{\circ}$ or $10^{\circ}$ greater than the preoperative angle between the inferior endplate of the UIV and the superior endplate of the UIV+2. ${ }^{8,26}$ It has been shown that one mechanism by which PJK occurs is fixation failure at the bone-implant interface, resulting in proximal screw loosening. ${ }^{3,4,17}$ Recent studies have shown that HU values measured on preoperative CT are independent predictors of implant loosening, and lower HU values are significantly correlated with a higher risk of pedicle screw loosening after lumbar fusion. ${ }^{34-36}$ Schwaiger et al. demonstrated that $\mathrm{HU}$ values on $\mathrm{CT}$ adequately differentiated patients with and without osteoporotic fractures, and they showed that HU values could predict pedicle screw loosening after fusion. ${ }^{36}$ However, previous studies have focused on the lumbar spine, establishing thresholds of 135 $\mathrm{HU}$ for osteopenic spines, $120 \mathrm{HU}$ for the risk of pedicle screw loosening, $110 \mathrm{HU}$ for osteoporotic spines, and 90 HU for the risk of vertebral body fracture. ${ }^{32,37,38}$ Although these numbers may be useful in identifying osteoporosis in patients undergoing spinal surgery for degenerative pathology, the same correlation has not been clearly shown in patients undergoing ASD correction, especially at the UIV in the thoracic spine. ${ }^{39}$ Thus, in ASD patients, the HUs of the LT spine may be more critical than the HUs of the lumbar spine. Although the reports on HU values at the UIV in PJK cases have been rare, Uei et al. evaluated the HUs at the UIV vertebral bodies in their series of ASD patients. ${ }^{18}$ They concluded that the mean BMD of vertebral bodies at T8 and T9 was significantly lower in elderly patients who required revision surgery. With regard to their study compared to ours, there are some differences. First, Uei and colleagues studied UIV levels from $\mathrm{T} 8$ to L2, incorporating the HU values of the lumbar spine and not focusing exclusively on the LT spine. Our study evaluated only the UIVs of the LT spine (T9 to T12) without any lumbar spine involvement. Second, they measured HU values from T8 to S1, and they did not focus their findings specifically on the UIV as we did. Despite these differences, however, our findings corroborate those of Uei et al., noting that decreased HUs on CT is associated with PJK.

Regardless of these findings, PJK remains a multifactorial phenomenon. Some possible reasons for PJK are long fusions to the sacrum, overcorrection of sagittal plane deformities, poor bone density, disruption of the posterior ligamentous tissues, or extreme rod stiffness. Although we have classified the types of PJK into ligamentous, boneimplant interface, or fracture, there may be multiple reasons for PJK, and they may not be clearly elucidated from the data. Although we controlled for PI-LL mismatch and SVA, there were patients who had increased TPA and PT, indicating that there was probably a higher proportion of patients who did have some degree of imbalance in addition to the lower HU values.

As mentioned above, previous studies have reported $\mathrm{HU}$ thresholds of $135 \mathrm{HU}$ for osteopenic spines, $120 \mathrm{HU}$ for the risk of pedicle screw loosening, $110 \mathrm{HU}$ for osteoporotic spines, and $90 \mathrm{HU}$ for the risk of vertebral body fracture. However, these studies have not specifically evaluated the HU threshold for PJK in ASD patients, nor did they evaluate HU thresholds specifically in the thoracic spine. In our study, we found that the optimal preoperative HU at the UIV cutoff value determined by the Youden index was $104 \mathrm{HU}$ (sensitivity 0.840 , specificity 0.517 ; Fig. 2). Given this information, our data reflect that the preoperative LT spine HU value of less than $104 \mathrm{HU}$ may be a risk factor for PJK after ASD correction. In addition, our results suggest that preoperative HUs at the UIV, UIV+1, and UIV+2 were significantly lower in the patients with PJK than in those without $(\mathrm{p}=0.011,0.02$, and 0.018 , respectively). Thus, the HUs at the UIV, UIV+1, and UIV+2 may be specifically associated with PJK. One point of note is that the Youden index was fairly low, but patients who demonstrated PJK had higher values. One reason is that there was a large range of HU values in the cohort with PJK. Because of this, a low cutoff was used, although some patients with PJK had higher HU values. It must also be kept in mind that per The International Society for Clinical Densitometry position statement, it is important to ensure that these HU values are valid "only if validated machine-specific cutoff values and scanner stability have been established" (https://www.iscd.org/ official-positions/).

With regard to clinical management, patients who have suspiciously low HU values on CT are sent for DXA to formally evaluate their bone density. If they are osteoporotic, their surgery is delayed, and they are placed on teriparatide or another medication for 3-6 months. If the patient is osteopenic (T-score $<-2.0$ ), they will generally have their surgery postponed, and they will be placed on teriparatide if they will be undergoing a large, corrective surgical procedure. However, if the patient is in severe, disabling pain and the surgery will not involve tremendous corrective forces but only a stabilization procedure, then they may be considered to proceed with surgery and given medication after surgery. Generally, the surgical UIV is not taken up into the upper thoracic spine unless there is significant TK, but this is considered as an option in patients with poor bone density.

There are several limitations to this study. First, it is a single-center retrospective study with a relatively small number of patients. Second, the presence of osteotomies (three column or posterior column) was not matched for because of the relatively small number of patients. Such a delineation would have probably made the study underpowered if the patients were divided into these two different groups. However, our data should be interpreted with the understanding that it has been shown that significant sagittal correction is associated with significant stress to the proximal adjacent disc and vertebrae. ${ }^{3}$ Nonetheless, we did not find a difference between the two groups with regard to pre- and postoperative PI-LL mismatch and SVA, indicating that the corrective magnitude in the sagittal plane between the two groups was not different. However, the PT and TPA were different, indicating that there may still have been some sagittal parameter that acted as a confounding variable with these data. Another limitation to this study that should be considered is the number of patients who were excluded, which may have led to selection bias. Despite these limitations, however, we did find statistical differences in HUs between the PJK and non- 
PJK cohorts, which is consistent with previous studies that have shown that a decreased HU is associated with both PJK and implant failure.

\section{Conclusions}

In patients undergoing sagittal correction with fusions from the LT spine to the sacrum for ASD, PJK was associated with lower HU values in the LT spine at the UIV, $\mathrm{UIV}+1$, and $\mathrm{UIV+2}$. The measurement of $\mathrm{HU}$ values on preoperative CTs may be a useful adjunct when planning surgical correction of ASD.

\section{Acknowledgments}

We are grateful for the financial support from the National Science Foundation of China (grant no. 81401790), Natural Science Foundation of Jiangxi Province (grant no. 20171ACB21057), and Science Research Project of Jiangxi Provincial Department of Education (GJJ160028).

\section{References}

1. Acaroglu E, Guler UO, Olgun ZD, et al. Multiple regression analysis of factors affecting health-related quality of life in adult spinal deformity. Spine Deform. 2015;3(4):360-366.

2. Schwab F, Dubey A, Gamez L, et al. Adult scoliosis: prevalence, SF-36, and nutritional parameters in an elderly volunteer population. Spine (Phila Pa 1976). 2005;30(9): 1082-1085.

3. Maruo K, Ha Y, Inoue S, et al. Predictive factors for proximal junctional kyphosis in long fusions to the sacrum in adult spinal deformity. Spine (Phila Pa 1976). 2013;38(23):E1469E1476.

4. Kim YC, Lenke LG, Bridwell KH, et al. Results of revision surgery for proximal junctional kyphosis following posterior segmental instrumentation: minimum 2-year postrevision follow-up. Spine (Phila Pa 1976). 2016;41(24):E1444-E1452.

5. Smith MW, Annis P, Lawrence BD, et al. Acute proximal junctional failure in patients with preoperative sagittal imbalance. Spine J. 2015;15(10):2142-2148.

6. Hostin R, McCarthy I, O'Brien M, et al. Incidence, mode, and location of acute proximal junctional failures after surgical treatment of adult spinal deformity. Spine (Phila Pa 1976). 2013;38(12):1008-1015.

7. Protopsaltis TS, Diebo BG, Lafage R, et al. Identifying thoracic compensation and predicting reciprocal thoracic kyphosis and proximal junctional kyphosis in adult spinal deformity surgery. Spine (Phila Pa 1976). 2018;43(21):1479-1486.

8. Glattes RC, Bridwell KH, Lenke LG, et al. Proximal junctional kyphosis in adult spinal deformity following long instrumented posterior spinal fusion: incidence, outcomes, and risk factor analysis. Spine (Phila Pa 1976). 2005;30(14): 1643-1649.

9. Kim HJ, Bridwell KH, Lenke LG, et al. Patients with proximal junctional kyphosis requiring revision surgery have higher postoperative lumbar lordosis and larger sagittal balance corrections. Spine (Phila Pa 1976). 2014;39(9):E576-E580.

10. Nicholls FH, Bae J, Theologis AA, et al. Factors associated with the development of and revision for proximal junctional kyphosis in 440 consecutive adult spinal deformity patients. Spine (Phila Pa 1976). 2017;42(22):1693-1698.

11. Kim YJ, Bridwell KH, Lenke LG, et al. Proximal junctional kyphosis in adult spinal deformity after segmental posterior spinal instrumentation and fusion: minimum five-year follow-up. Spine (Phila Pa 1976). 2008;33(20):2179-2184.

12. Viswanathan VK, Kukreja S, Minnema AJ, Farhadi HF. Prospective assessment of the safety and early outcomes of sublaminar band placement for the prevention of proximal junctional kyphosis. J Neurosurg Spine. 2018;28(5):520-531.

13. Lewis SJ, Abbas H, Chua S, et al. Upper instrumented vertebral fractures in long lumbar fusions: what are the associated risk factors? Spine (Phila Pa 1976). 2012;37(16):1407-1414.

14. Theologis AA, Burch S. Prevention of acute proximal junctional fractures after long thoracolumbar posterior fusions for adult spinal deformity using 2-level cement augmentation at the upper instrumented vertebra and the vertebra 1 level proximal to the upper instrumented vertebra. Spine (Phila $\mathrm{Pa}$ 1976). 2015;40(19):1516-1526.

15. Yagi M, Akilah KB, Boachie-Adjei O. Incidence, risk factors and classification of proximal junctional kyphosis: surgical outcomes review of adult idiopathic scoliosis. Spine (Phila Pa 1976). 2011;36(1):E60-E68.

16. Hart R, McCarthy I, O'Brien M, et al. Identification of decision criteria for revision surgery among patients with proximal junctional failure after surgical treatment of spinal deformity. Spine (Phila Pa 1976). 2013;38(19):E1223-E1227.

17. Hyun SJ, Lee BH, Park JH, et al. Proximal junctional kyphosis and proximal junctional failure following adult spinal deformity surgery. Korean J Spine. 2017;14(4):126-132.

18. Uei H, Tokuhashi Y, Maseda M, et al. Exploratory analysis of predictors of revision surgery for proximal junctional kyphosis or additional postoperative vertebral fracture following adult spinal deformity surgery in elderly patients: a retrospective cohort study. J Orthop Surg Res. 2018;13(1):252.

19. Yagi M, King AB, Boachie-Adjei O. Incidence, risk factors, and natural course of proximal junctional kyphosis: surgical outcomes review of adult idiopathic scoliosis. Minimum 5 years of follow-up. Spine (Phila Pa 1976). 2012;37(17): 1479-1489.

20. Yagi M, Fujita N, Tsuji O, et al. Low bone-mineral density is a significant risk for proximal junctional failure after surgical correction of adult spinal deformity: a propensity score-matched analysis. Spine (Phila Pa 1976). 2018;43(7): 485-491.

21. Watanabe K, Lenke LG, Bridwell KH, et al. Proximal junctional vertebral fracture in adults after spinal deformity surgery using pedicle screw constructs: analysis of morphological features. Spine (Phila Pa 1976). 2010;35(2):138-145.

22. Demir Ö, Öksüz E, Deniz FE, Demir O. Assessing the effects of lumbar posterior stabilization and fusion to vertebral bone density in stabilized and adjacent segments by using Hounsfield unit. J Spine Surg. 2017;3(4):548-553.

23. Schreiber JJ, Anderson PA, Hsu WK. Use of computed tomography for assessing bone mineral density. Neurosurg Focus. 2014;37(1):E4.

24. Gausden EB, Nwachukwu BU, Schreiber JJ, et al. Opportunistic use of CT imaging for osteoporosis screening and bone density assessment: a qualitative systematic review. J Bone Joint Surg Am. 2017;99(18):1580-1590.

25. Schreiber JJ, Anderson PA, Rosas HG, et al. Hounsfield units for assessing bone mineral density and strength: a tool for osteoporosis management. J Bone Joint Surg Am. 2011;93(11): 1057-1063.

26. Mummaneni PV, Park P, Fu KM, et al. Does minimally invasive percutaneous posterior instrumentation reduce risk of proximal junctional kyphosis in adult spinal deformity surgery? A propensity-matched cohort analysis. Neurosurgery. 2016;78(1):101-108.

27. Burch S, Feldstein M, Hoffmann PF, Keaveny TM. Prevalence of poor bone quality in women undergoing spinal fusion using biomechanical-CT analysis. Spine (Phila Pa 1976). 2016;41(3):246-252.

28. Hendrickson NR, Pickhardt PJ, Del Rio AM, et al. Bone mineral density $\mathrm{T}$-scores derived from $\mathrm{CT}$ attenuation numbers (Hounsfield units): clinical utility and correlation with dualenergy x-ray absorptiometry. Iowa Orthop J. 2018;38:25-31. 
29. Lewiecki EM, Keaveny TM, Kopperdahl DL, et al. Oncemonthly oral ibandronate improves biomechanical determinants of bone strength in women with postmenopausal osteoporosis. J Clin Endocrinol Metab. 2009;94(1):171-180.

30. Lee S, Chung CK, Oh SH, Park SB. Correlation between bone mineral density measured by dual-energy x-ray absorptiometry and Hounsfield units measured by diagnostic CT in lumbar spine. J Korean Neurosurg Soc. 2013;54(5):384-389.

31. Choi MK, Kim SM, Lim JK. Diagnostic efficacy of Hounsfield units in spine CT for the assessment of real bone mineral density of degenerative spine: correlation study between T-scores determined by DEXA scan and Hounsfield units from CT. Acta Neurochir (Wien). 2016;158(7):1421-1427.

32. Anderson PA, Polly DW, Binkley NC, Pickhardt PJ. Clinical use of opportunistic computed tomography screening for osteoporosis. J Bone Joint Surg Am. 2018;100(23):2073-2081.

33. Kadri A, Binkley N, Hare KJ, Anderson PA. Bone health optimization in orthopaedic surgery. J Bone Joint Surg Am. 2020;102(7):574-581.

34. Zou D, Muheremu A, Sun Z, et al. Computed tomography Hounsfield unit-based prediction of pedicle screw loosening after surgery for degenerative lumbar spine disease. J Neurosurg Spine. 2020;32(5):716-721.

35. Bredow J, Boese CK, Werner CM, et al. Predictive validity of preoperative CT scans and the risk of pedicle screw loosening in spinal surgery. Arch Orthop Trauma Surg. 2016;136(8): 1063-1067.

36. Schwaiger BJ, Gersing AS, Baum T, et al. Bone mineral density values derived from routine lumbar spine multidetector row CT predict osteoporotic vertebral fractures and screw loosening. AJNR Am J Neuroradiol. 2014;35(8):1628-1633.

37. Zaidi Q, Danisa OA, Cheng W. Measurement techniques and utility of Hounsfield unit values for assessment of bone quality prior to spinal instrumentation: a review of current literature. Spine (Phila Pa 1976). 2019;44(4):E239-E244.

38. Graffy PM, Lee SJ, Ziemlewicz TJ, Pickhardt PJ. Prevalence of vertebral compression fractures on routine CT scans according to L1 trabecular attenuation: determining relevant thresholds for opportunistic osteoporosis screening. AJR Am J Roentgenol. 2017;209(3):491-496.

39. Kohan EM, Nemani VM, Hershman S, et al. Lumbar computed tomography scans are not appropriate surrogates for bone mineral density scans in primary adult spinal deformity. Neurosurg Focus. 2017;43(6):E4.

\section{Disclosures}

Dr. Mummaneni is a consultant for Globus, DePuy Synthes, and Stryker; receives honoraria from Spineart; receives royalties from DePuy Synthes, Thieme Publishing, and Springer Publishing; has direct stock ownership in Spinicity ISD; and receives support from AO Spine, NREF, and ISSG for non-study-related clinical or research effort. Dr. Ames receives royalties from Stryker, Biomet Zimmer Spine, DePuy Synthes Spine, Next Orthosurgical, $\mathrm{K} 2 \mathrm{M}$, Medicrea, and NuVasive; is a consultant for Medtronic, Medicrea, K2M, Globus, and DePuy Synthes; conducts research for Titan Spine, DePuy Synthes, and ISSG; serves on the editorial board of Operative Neurosurgery; receives grant funding from SRS; serves on the executive committee for ISSG; and serves as a director for Global Spinal Analytics. Dr. Burch is a consultant for Medtronic and Globus. Dr. Berven has ownership of GreenSun Medical and Providence Medical; is a consultant for Medtronic, Stryker, Globus, Integrity Spine, Innovasis, and Medicrea; receives royalties from Medtronic and Stryker; and has received grants from AO Spine, NIH, and NSF. Dr. Chou is a consultant for Medtronic and Globus and receives royalties from Globus.

\section{Author Contributions}

Conception and design: Chou. Acquisition of data: Duan, Rivera, Guinn, Wang, Xi, Li, Wu. Analysis and interpretation of data: Duan. Drafting the article: Duan. Critically revising the article: Mummaneni, Burch, Berven, Chou. Reviewed submitted version of manuscript: Chou. Approved the final version of the manuscript on behalf of all authors: Duan. Statistical analysis: Duan. Administrative/technical/material support: Mummaneni, Rivera, Ames, Burch, Berven, Chou. Study supervision: Chou.

\section{Correspondence}

Ping-Guo Duan: University of California, San Francisco, CA. pink1198@163.com. 